ESAIM: PROCEEDINGS, May 2009, Vol. 27, p. 240-253

C. Besse, O. Goubet, T. Goudon \& S. Nicaise, Editors

\title{
A NUMERICAL APPROACH FOR MODELLING THIN CRACKED PLATES WITH XFEM
}

\author{
JÉRÉMIE LASRY ${ }^{1}$, YVES RENARD ${ }^{2}$ AND MICHEL SALAÜN ${ }^{3}$
}

\begin{abstract}
The modelization of bending plates with through the thickness cracks is investigated. We consider the Kirchhoff-Love plate model which is valid for very thin plates. We apply the eXtended Finite Element Method (XFEM) strategy: enrichment of the finite element space with the asymptotic bending and with the discontinuity across the crack. We present two variants and their numerical validations and also a numerical computation of the stress intensity factors.
\end{abstract}

Résumé. Cet article traite de l'approximation numérique des plaques en flexion en présence d'une fissure traversante. Nous considérons un modèle de Kirchhoff-Love, valide pour les plaques très minces, et des éléments finis de type triangles HCT et quadrangles FVS réduits. La stratégie de la méthode des éléments finis étendue (XFEM) est alors appliquée : enrichissement de l'espace élément fini par le déplacement de flexion asymptotique en fond de fissure et par la discontinuité à la traversée de la fissure. Nous présentons deux variantes que nous validons numériquement ainsi que des calculs de facteurs d'intensité de contrainte.

\section{INTRODUCTION}

Very thin plates are widely used for instance in aircraft structures and some of them may present through the thickness cracks. We describe in this paper an adaptation of XFEM (eXtended Finite Element Method) to the computation of thin cracked plates. XFEM is a strategy initially developed for plane elasticity cracked problems (see [16,17]) and is now the subject of a wide literature (among many others, see $[2-4,12,18,20]$ and references therein). It mainly consists in the introduction of the discontinuity across the crack and of the asymptotic displacements into the finite element space. As far as we know, the unique attempt to adapt XFEM to plate models is presented in [10] in which a Mindlin-Reissner model is considered. However, in this reference, the numerical tests are degraded by an important shear locking effect for very thin plates, despite the use of some classical locking-free elements. This suggests that the locking effect is due to the XFEM enrichment.

Even though most of the finite element codes are based on the Mindlin-Reissner plate model, the socalled Kirchhoff-Love model provides also a realistic description of the displacement for a thin plate since it is the limit model of the three-dimensional elasticity model when the thickness vanishes (see [7]). For instance, the panels used in aeronautic structures can be about one millimeter thin, for several meters long. On this kind of plate, the shear effect can generally be neglected and consequently the Kirchhoff-Love model is mechanically appropriate. Furthermore, for through the thickness cracks, the limit of the energy release rate of the three-dimensional model can be expressed with the Kirchhoff-Love model solution (see [8] and [9]).

\footnotetext{
${ }^{1}$ MIP-IMT, CNRS UMR 5219, INSAT, Complexe scientifique de Rangueil, 31077 Toulouse, France, j_lasry@insa-toulouse.fr

2 Université de Lyon, CNRS

INSA-Lyon, ICJ UMR5208, LaMCoS UMR5259, F-69621, Villeurbanne, France, yves.renard@insa-lyon.fr

${ }^{3}$ Université de Toulouse, ISAE, 10 av. Edouard Belin, 31055 Toulouse cedex, France, Michel.Salaun@isae.fr
} 
The Kirchhoff-Love model is not submitted to the so-called "shear locking" phenomenon for the finite element discretization, even for a very small thickness. So, the reliability of the numerical results is potentially better compared to the use of the Mindlin-Reissner model, which needs some specific treatments to avoid the locking phenomenon such as a reduced integration (QUAD 4 element, see [15]). Moreover, these treatments are not straightforwardly adaptable to an enriched finite element space.

Since the Kirchhoff-Love model corresponds to a fourth order partial differential equation, a conformal finite element method needs the use of $\mathscr{C}^{1}$ (continuously differentiable) elements. We consider the reduced Hsieh-Clough-Tocher triangle (reduced HCT) and the reduced Fraejis de Veubeke-Sanders quadrilateral (reduced FVS) because they are the less costly conformal $\mathscr{C}^{1}$ elements (piecewise cubic elements), see [5]. In the XFEM framework, the knowledge of the asymptotic crack tip displacement is required. For a Kirchhoff-Love isotropic plate, it corresponds to the bilaplacian singularities (see [11]).

Now, we briefly describe the features of the specific XFEM enrichment. In order to represent the discontinuity across the crack, a "jump function" (or Heaviside function) is used. Then two strategies for the crack tip enrichment are proposed, following the ideas already presented in [12]. In both of them, an enrichment area of fixed size is defined, centered on the crack tip. In the first strategy, the crack tip asymptotic displacements are added to the finite element space multiplied by the shape functions corresponding to the nodes which are in the enrichment area. In the second one, the asymptotic displacements are added without being multiplied by some shape functions. In order to limit its influence, this enrichement is only considered inside the enrichment area. A matching condition is prescribed in order to ensure the (weak) continuity of the displacement and its derivatives across the interface between the enrichment area and the rest of the domain.

The paper is organized as follows. Section 1 describes the model problem. Section 2 is devoted to the finite element discretization of the Kirchhoff-Love model. In Section 3, the two enrichment strategies are detailed. In section 4, some numerical results are presented, which illustrate the capabilities of these methods. The last section deals with the computation of the Stress Intensity Factors: these values are used in the crack propagation criteria by mechanical engineers.

\section{The Model Problem}

\subsection{Notations and variational formulation}

Let us consider a thin plate, i.e. a plane structure for which one dimension, called the thickness, is very small compared to the others. For this kind of structures, starting from a priori hypotheses on the expression of the displacement fields, a two-dimensional problem is usually derived from the three-dimensional elasticity formulation by means of integration along the thickness. Then, the unknown variables are set down on the mid-plane of the plate, denoted by $\omega$.

The mid-plane $\omega$ is an open subset of $\mathbb{R}^{2}$. In the three-dimensional cartesian referential, the plate occupy the space

$$
\left\{\left(x_{1}, x_{2}, x_{3}\right) \in \mathbb{R}^{3},\left(x_{1}, x_{2}\right) \in \omega \text { and } x_{3} \in\right]-\varepsilon ; \varepsilon[\} .
$$

So, the $x_{3}$ coordinate corresponds to the transverse direction, and all the mid-plane points have their third coordinate equal to 0 . The thickness is $2 \varepsilon$ (see Fig. 1). Finally, we assume that the plate has a through the thickness crack and that the material is isotropic, of Young's modulus $E$ and Poisson's ratio $\nu$.

In plate theory, the following approximation of the three-dimensional displacements is usually considered:

$$
\left\{\begin{array}{l}
u_{1}\left(x_{1}, x_{2}, x_{3}\right)=\bar{u}_{1}\left(x_{1}, x_{2}\right)+x_{3} \phi_{1}\left(x_{1}, x_{2}\right), \\
u_{2}\left(x_{1}, x_{2}, x_{3}\right)=\bar{u}_{2}\left(x_{1}, x_{2}\right)+x_{3} \phi_{2}\left(x_{1}, x_{2}\right), \\
u_{3}\left(x_{1}, x_{2}, x_{3}\right)=u_{3}\left(x_{1}, x_{2}\right) .
\end{array}\right.
$$

In these expressions, $\bar{u}_{1}$ and $\bar{u}_{2}$ are the membrane displacements of the mid-plane points while $u_{3}$ is the deflection, $\phi_{1}$ and $\phi_{2}$ are the section rotations. In the case of an isotropic material, the variational formulation splits into two independent problems: the first, called the membrane problem, deals only with the 


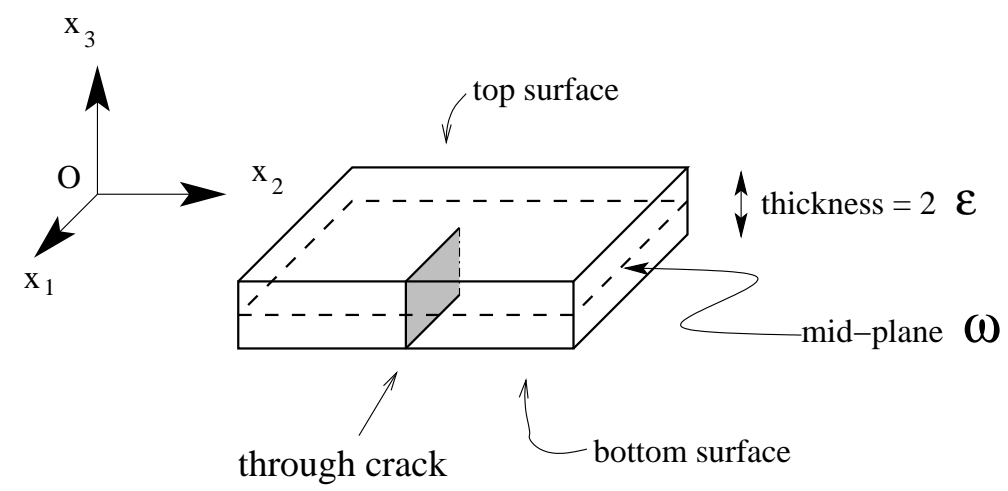

FIGURE 1. The cracked thin plate. The thickness is oversized for the sake of clarity

membrane displacements, while the second, called the bending problem, concerns the deflection and the rotations. The membrane problem corresponds to the classical plane elasticity problem and has been already treated in many references (see for instance $[3,12]$ ). So, here, we only consider the bending problem.

In industrial finite element codes, the most widely used plate model is the Mindlin-Reissner one, for which the displacement is given by (1). Nevertheless, for reasons mentioned in the introduction, we choose here to work with the Kirchhoff-Love model, which can be seen as a particular case of (1), as it is obtained by introducing the Kirchhoff-Love assumptions, which read

$$
\nabla u_{3}+\phi=0 \text { i.e. }\left\{\begin{array}{l}
\phi_{1}=-\partial_{1} u_{3} \\
\phi_{2}=-\partial_{2} u_{3}
\end{array}\right.
$$

where the notation $\partial_{\alpha}$ stands for the partial derivative with respect to $x_{\alpha}$. A first consequence of this relation is that the transverse shear strain is identically zero, which avoids the shear locking problem. A second consequence of (2) is that the section rotation only depends on the transverse displacement. It means that this displacement is the only unknown function for the bending problem. For convenience, it will be denoted by $u$ in the following. So, in the Kirchhoff-Love framework and for a pure bending problem, the three-dimensional displacement reads

$$
\left\{\begin{array}{l}
u_{1}\left(x_{1}, x_{2}, x_{3}\right)=-x_{3} \partial_{1} u\left(x_{1}, x_{2}\right), \\
u_{2}\left(x_{1}, x_{2}, x_{3}\right)=-x_{3} \partial_{2} u\left(x_{1}, x_{2}\right), \\
u_{3}\left(x_{1}, x_{2}, x_{3}\right)=u\left(x_{1}, x_{2}\right)
\end{array}\right.
$$

For the sake of simplicity, we assume the plate is clamped on its boundary and the crack faces are traction free. Then, the plate is subjected to a volume force, say $f$ of coordinates $\left(f_{1}, f_{2}, f_{3}\right)$, and two surface forces, say $g^{+}$and $g^{-}$, applied on the top and bottom surfaces. The variational formulation (or virtual work formulation) of the Kirchhoff-Love model reads as

$$
\left\{\begin{array}{l}
\text { Find } u \in H_{0}^{2}(\omega) \text { such that for any } v \in H_{0}^{2}(\omega) \\
\int_{\omega} \frac{2 E \varepsilon^{3}}{3\left(1-\nu^{2}\right)}\left[(1-\nu) \partial_{\alpha \beta}^{2} u+\nu \Delta u \delta_{\alpha \beta}\right] \partial_{\alpha \beta}^{2} v d x=\int_{\omega}\left[F v+M_{\alpha} \partial_{\alpha} v\right] d x
\end{array}\right.
$$

where:

$-F=\int_{-\varepsilon}^{\varepsilon} f_{3} d x_{3}+g_{3}^{+}+g_{3}^{-}$, which is the resulting transverse loading,

- $M_{\alpha}=\int_{-\varepsilon}^{\varepsilon} x_{3} f_{\alpha} d x_{3}+\varepsilon\left(g_{\alpha}^{+}-g_{\alpha}^{-}\right)$, which is the resulting moment loading.

Moreover $\delta_{\alpha \beta}$ stands for the Kronecker's symbol and the summation convention over repeated indices is adopted, Greek indices varying in $\{1,2\}$. Finally, $H_{0}^{2}(\omega)$ is the classical Sobolev space of square integrable functions whose first and second derivatives in the distributions sense are square integrable, and which vanish on the boundary, like their normal derivative (see [1] for instance). 


\subsection{Asymptotic displacement near the crack tip and Fracture Modes}

In the Kirchhoff-Love plate model, there are two fracture modes. Applying a symmetric bending leads to the first fracture mode, while applying an anti-symmetric bending or a transverse shear, leads to the second one (see Fig. 2).

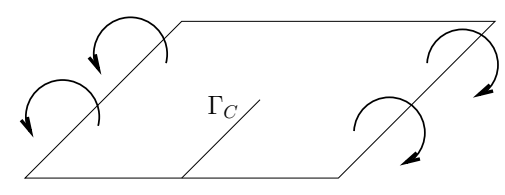

Mode I

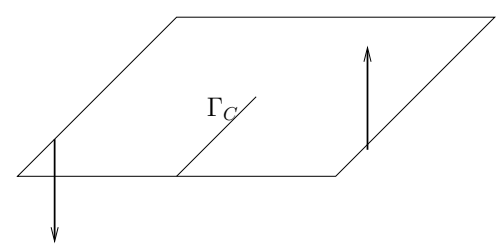

Mode II

FIGURE 2. Fracture modes for Kirchhoff-Love bending model ( $\Gamma_{C}$ is the crack). Left: a symmetric bending leads to mode I. Right: a shear bending leads to mode II.

To characterize them, let us recall that the governing equation related to the bending variational problem (3) reads

$$
\frac{2 E \varepsilon^{3}}{3\left(1-\nu^{2}\right)} \Delta^{2} u=F+\partial_{\alpha} M_{\alpha}
$$

on the mid-plane $\omega$. It is a bilaplacian problem for which the singularities are well-known (see [11]). So, close to the crack tip, the displacement may be written as $u=u_{r}+u_{s}$, where $u_{r}$ stands for the regular part of the transverse displacement and belongs to $H^{3}(\omega)$. The singular part $u_{s}$ reads

$u_{s}(r, \theta)=\frac{\sqrt{2}}{2} \frac{1-\nu^{2}}{E \varepsilon(3+\nu)} r^{3 / 2}\left[K_{1}\left(\frac{\nu+7}{3(\nu-1)} \cos \frac{3}{2} \theta+\cos \frac{\theta}{2}\right)+K_{2}\left(\frac{3 \nu+5}{3(\nu-1)} \sin \frac{3}{2} \theta+\sin \frac{\theta}{2}\right)\right]$

in polar coordinates relatively to the crack tip (see Fig. 3). Let us recall that this singular displacement belongs to $H^{5 / 2-\eta}(\omega)$ for any $\eta>0$.

The scalar coefficients $K_{1}$ and $K_{2}$ are the so-called "Stress Intensity Factors". They are widely used in fracture mechanics for crack propagation.

We recall that the Kirchhoff-Love plate theory corresponds to the limit of the three-dimensional elasticity theory, when the thickness vanishes. However, the singularities we present here are deduced from the Kirchhoff-Love theory, and not from the three-dimensional elasticity theory. The reader interested by the link between the singularities of these two theories is referred to the numerical study [21].

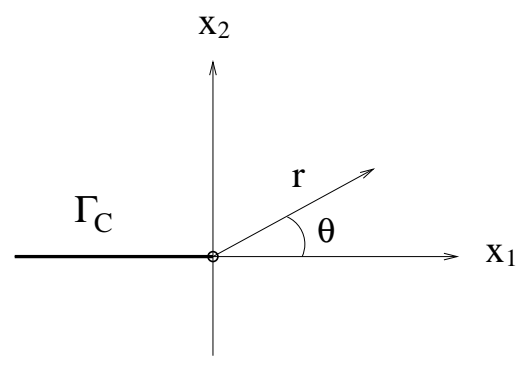

FIGURE 3. System of polar coordinates relatively to the crack tip (the crack is in bold line).

\section{Finite ElEMENT APPROXIMATION OF THE KiRCHHOFF-LOVE MODEL}

Let us introduce now the finite element discretization of the variational formulation (3). In order to have a conformal method, the finite element space $V^{h}$ has to satisfy $V^{h} \subset H_{0}^{2}(\omega)$. This leads to the use of $\mathscr{C}^{1}$ 
finite elements. Among the available elements having this regularity, the reduced HCT triangles (see [5], p. 356-357) and FVS quadrangles (see [5], p. 359-360) are of particular interest: they are the less costly elements (see [5], p. 355). For both elements, the triangle (resp. quadrangle) is divided into three (resp. four) sub-triangles (see Fig. 4). The basis functions are $P_{3}$ polynomials on each sub-triangle and matched $\mathscr{C}^{1}$ across each internal edge. In addition, to decrease the number of dof (degrees of freedom), the normal derivative is assumed to vary linearly along the external edges of the elements (this assumption does not hold on the internal edges). At the end there are only three dof on each node for both elements: the value of the function and its first derivatives. So, these elements have the two following advantages:

(1) The computational cost is limited to three dof for each node of the mesh, like a classical MindlinReissner element (the deflection and the two section rotations).

(2) The theoretical error is in $O(h)$ and $O\left(h^{2}\right)$ for the $H^{2}$ and $L^{2}$ norm (respectively), on regular problems ( $h$ stands for the mesh parameter). The minimum regularity assumption for this error estimate to hold is that the exact solution belongs to $H^{3}(\omega)$ (see [6]).

So, the reduced HCT or FVS elements and standard Mindlin elements have the same features as far as numerical cost and accuracy are concerned.
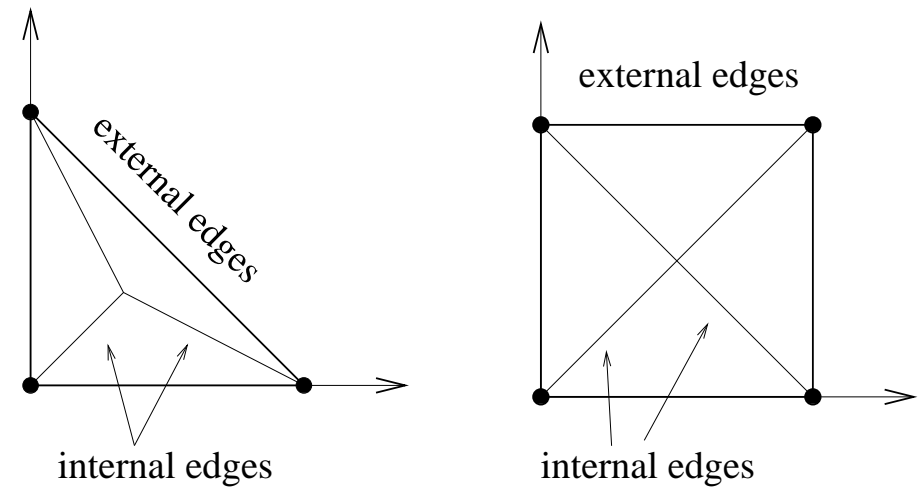

FIGURE 4. HCT triangle and FVS quadrangle. Location of degrees of freedom and sub-triangles.

\section{XFEM ENRICHMENTS}

To define the XFEM enrichment strategies, we follow the ideas presented in previous papers [3,12]. The discontinuity of the displacement across the crack is represented using a Heaviside-like function, which is multiplied by the finite element shape functions. For the nonsmooth enrichment close to the crack tip, we propose two strategies. In both of them, an enrichment area of fixed size is defined and the nonsmooth functions are added inside all this area. Then, the unknown function may be written as

$$
u^{h}=\sum_{i} a_{i} \varphi_{i}+\sum_{j \in J} b_{j} H \varphi_{j}+\sum_{k \in K} c_{k} f_{k},
$$

where $\varphi_{j}$ are the basis functions of the reduced HCT/FVS elements. The jump of the $H$ function is located on the crack; the set $J$ denotes the degrees of freedom whose shape function support is completely crossed by the crack (see Fig. 5). The exact meaning and expressions of the set $K$ and functions $f_{k}$ will be detailed in sub-sections 3.2 and 3.3 for the two strategies.

\subsection{An incompatibility between H-enrichment and FVS element}

Before focusing on the singular enrichment, let us describe a numerical difficulty and one way to overcome it. It appears an incompatibility between the Heaviside-like enrichment and the FVS element, reduced or not. If a straight crack crosses two adjacent edges of a quadrangle, then a non-invertible linear system 


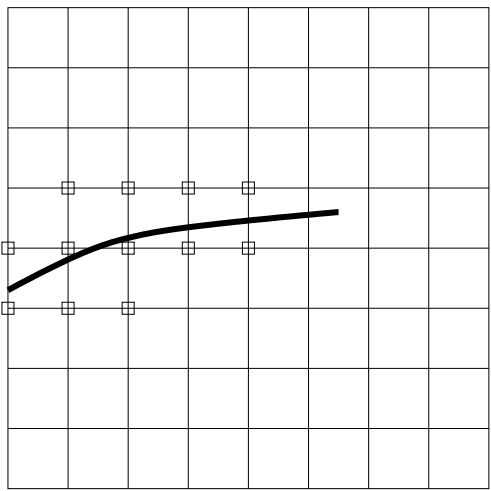

FIGURE 5. Set of nodes to be enriched along the crack.

may result. The reason is there exists a function, in the FVS functions space, that is completely zero on two adjacent sub-triangles of the quadrangle (see Fig. 6). Consequently, if the quadrangle is cut along those two sub-triangles (see Fig. 7, left), then the regular degrees of freedom associated with the opposite node are linear combinations of the $H$-enriched ones. This is not a proof that the resulting matrix will be singular, but at least an insight. In our practical applications, with not too coarse meshes, the linear system was always singular (an example of such mesh is given Fig. 8). In addition, the problem is independant of the distance between nodes and crack: whether they are far or not, the non-invertibility remains. Let us also remark that it is not particularly due to the fact that the mesh is structured or not, even if, in our tests, the problem arises only with non-structured meshes. By the way, in the case of a straight crack and structured meshes, the quadrangles had always three sub-triangles crossed by the crack (see Fig. 7, right), which does not cause any non-invertibility.

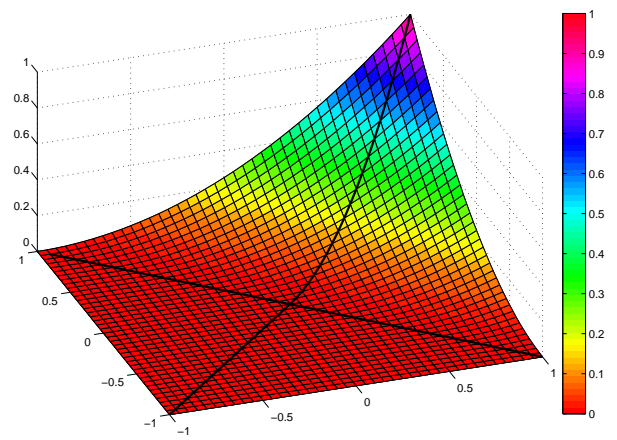

FIGURE 6. A function belonging to the FVS space functions. We can see that both the function and its derivatives are completely zero on two adjacent sub-triangles.

Let us also observe that this incompatibility does not occur with the reduced HCT triangle. Hence, to solve it on FVS, we have divided each quadrangle crossed by the crack in two triangles. It is an easy and quite cheap operation, and we emphasize on the fact that it is not a remeshing: the nodes of the "new" mesh are exactly the same as those of the "old" one.

\subsection{First enrichment strategy: adding degrees of freedom on each node}

The first strategy is directly inspired from the one presented in $[3,12]$ for plane elasticity problems. In these two references, this method was called respectively "geometrical enrichment" or "XFEM with fixed enrichment area". Every node included in a fixed area around the crack tip is enriched with the nonsmooth functions (see Fig. 9). So, at each enriched node, a set of two degrees of freedom is added for each degree of 

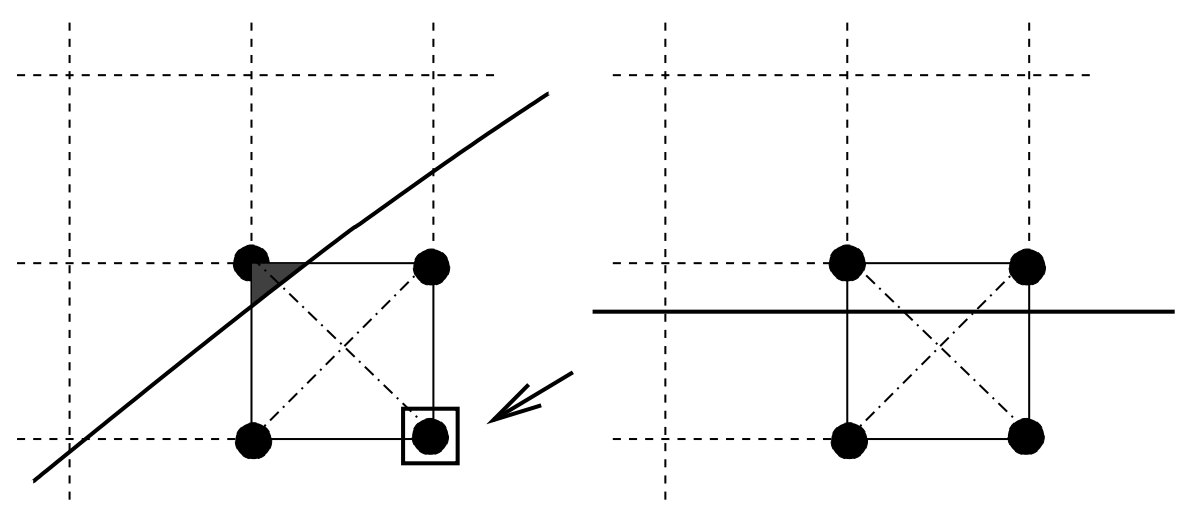

FIGURE 7. Left: two sub-triangles are crossed (usually leads to singular matrices). Right: three sub-triangles are crossed (always leads to regular systems).
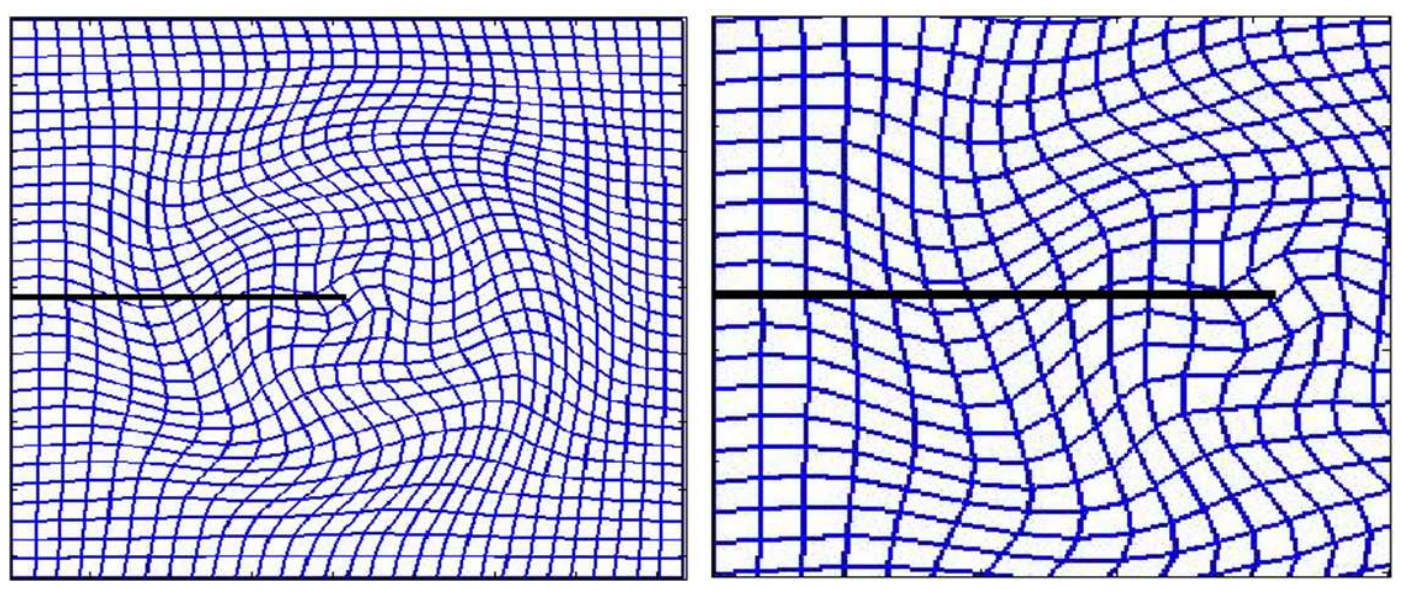

FIGURE 8. Left: example of mesh leading to a non-invertible linear system, with the crack (in black). Right: zoom on the crack.

freedom of the classical finite element method. This enrichment is obtained by multiplying the nonsmooth functions with the shape functions of the finite element. Then, the global unknown reads

$$
u^{h}=\sum_{i} a_{i} \varphi_{i}+\sum_{j \in J} b_{j} H \varphi_{j}+\sum_{k \in K} \sum_{l=1}^{2} c_{k l} F_{l} \varphi_{k}
$$

where $\left(F_{l}\right)_{l}$ are the nonsmooth functions, given by

$$
\begin{aligned}
& F_{1}=r^{3 / 2}\left(\frac{\nu+7}{3(\nu-1)} \cos \frac{3}{2} \theta+\cos \frac{\theta}{2}\right), \\
& F_{2}=r^{3 / 2}\left(\frac{3 \nu+5}{3(\nu-1)} \sin \frac{3}{2} \theta+\sin \frac{\theta}{2}\right) .
\end{aligned}
$$

in polar coordinates relatively to the crack tip (see Fig. 3). These functions are obviously associated with the singular part of the displacement as it is expressed in (5). Moreover, the set $K$ denotes the degrees of freedom associated with the nodes that are inside the enrichment zone: these nodes are indicated by a small circle on Fig. 9. As both reduced HCT and FVS elements have three degrees of freedom at each node, the addition of two nonsmooth functions means that six additional degrees of freedom are used for each node included in the nonsmooth enrichment area. Let us also remark that some nodes are enriched both with $H$ and nonsmooth functions. 


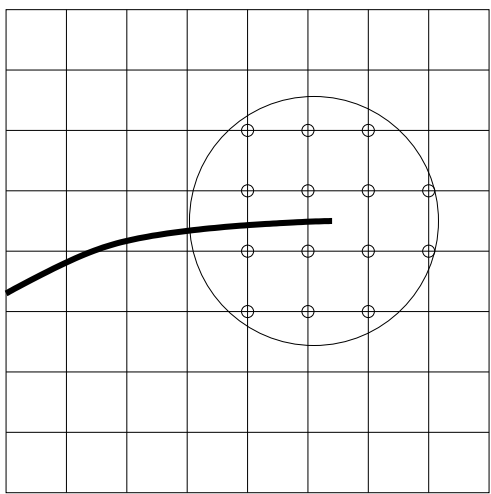

FIGURE 9. Set of nodes to be enriched with the nonsmooth functions.

\subsection{Second enrichment strategy: global nonsmooth functions and integral matching}

The second strategy is inspired by the so called "XFEM dof gathering with pointwise matching", introduced in [12]. Here, the idea is to reduce the number of degrees of freedom that are introduced in the finite element base. For this aim, the support of the singular added functions is the whole enrichment area (see Fig. 10) and they are not multiplied by the finite element basis functions. So, instead of 6 additional degrees of freedom per node inside the enrichment area, there are only 2 singular degrees of freedom for the whole system: the functions given by (7).

We introduce the boundary of the enrichment area, say $\Gamma$. It cuts $\omega$ into two sub-domains: the enrichment area, say $\omega_{1}$, and the rest of $\omega$, say $\omega_{2}$ (see Fig. 10). The unknowns defined on each domain $\omega_{i}$ are denoted by $u_{i}^{h}$ and their expressions read

$$
\left\{\begin{array}{l}
u_{1}^{h}=\sum_{i \in N_{1}} a_{i} \varphi_{i}+\sum_{j \in J_{1}} b_{j} H \varphi_{j}+\sum_{i=1}^{2} c_{i} F_{i} \\
u_{2}^{h}=\sum_{i \in N_{2}} a_{i} \varphi_{i}+\sum_{j \in J_{2}} b_{j} H \varphi_{j}
\end{array}\right.
$$

where $N_{1}$ and $N_{2}$ are the set of degrees of freedom that are located in $\omega_{1}$ and $\omega_{2}\left(N_{1} \cap N_{2}\right.$ is not empty: it corresponds to the set of nodes that are on the boundary $\Gamma$ ). In a same way, $J_{i}$ are the set of degrees of freedom of $J$ that are located in $\omega_{i}\left(J_{1} \cap J_{2}\right.$ is not empty for the same reason). Naturally, a matching condition is needed at the interface between the enrichment area and the rest of the domain, in order to insure the continuity of the function and its derivatives. The following relations were chosen at this aim

$$
\begin{aligned}
\int_{\Gamma} u_{1}^{h} \lambda & =\int_{\Gamma} u_{2}^{h} \lambda, \quad \forall \lambda \in \Lambda, \\
\int_{\Gamma} \partial_{n} u_{1}^{h} \mu & =\int_{\Gamma}-\partial_{n} u_{2}^{h} \mu, \quad \forall \mu \in M,
\end{aligned}
$$

where $\Lambda$ and $M$ are appropriate multiplier spaces. For example, it is possible to take $\Lambda$ as the space of piecewise polynomials of degree 2 and $M$ as piecewise polynomials of degree 1 . Let us observe the change of sign in front of the normal derivative $\partial_{n} u_{i}$ (second equation of (9)): it is due to the fact that the outside normal vector has an opposite sign whether it is used in $\partial_{n} u_{1}^{h}$ or $\partial_{n} u_{2}^{h}$.

\section{NUMERICAL EXPERIMENTS}

The numerical experiments that we present in this section have been performed with the open-source finite element library Getfem++ [19]. 


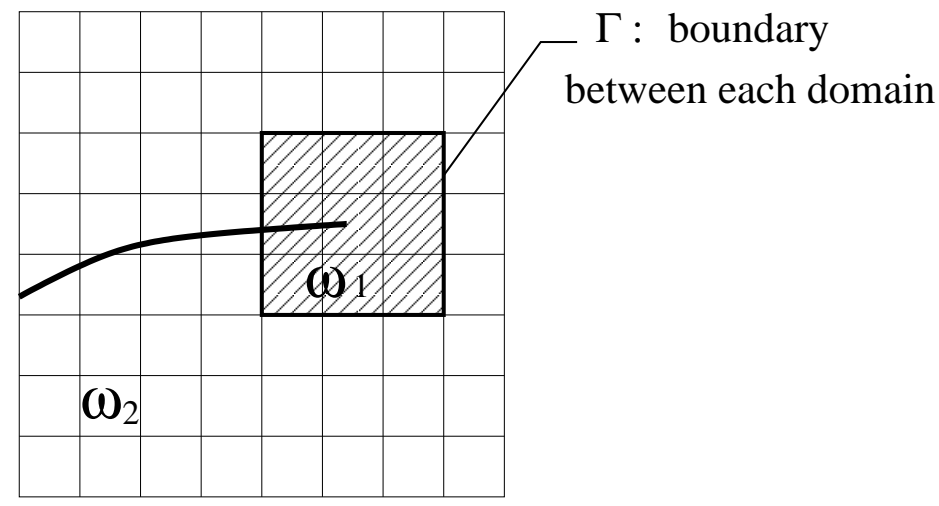

FIGURE 10. Set of elements which represents the support of the nonsmooth functions.

\subsection{Test problem}

To test various numerical methods, we define a benchmark problem: a plate with a straight through crack. The plate is a square, of edge 1. As mentioned before, the material is assumed to be isotropic, of Young's modulus $E$ and Poisson's ratio $\nu$. We took $E=14.98 \mathrm{kPa}, \nu=0.3$ and half the thickness $\varepsilon=0.045$ (however, with respect to numerical accuracy, the thickness is irrelevant). The exact solution of the problem is a pure mode II displacement, given by

$$
u^{e x}=\frac{1}{10} r^{3 / 2}\left(\frac{3 \nu+5}{3(\nu-1)} \sin \frac{3}{2} \theta+\sin \frac{\theta}{2}\right) .
$$

Of course, this is a solution of the equation $\frac{2 E \varepsilon^{3}}{3\left(1-\nu^{2}\right)} \Delta^{2} u=0$ with free-edge conditions on the crack. To conclude, let us just observe that, for this test problem, there is no volume nor surface forces: the loading is due to non-homogeneous boundary conditions, given by (10).

Our experiments consist in computing classical Finite Element Method (FEM) and XFEM solutions (denoted $u^{h}$ ), then to compute the $L^{2}$ and $H^{2}$ errors with respect to the exact solution $u$. We also pay attention to the rate of convergence: the number $\alpha$ such that $\left\|u^{h}-u\right\|_{V}=O\left(h^{\alpha}\right)$, with $V=L^{2}(\omega)$ or $H^{2}(\omega)$.

The coarsest mesh was made with 11 elements on each edge of the domain, and the more refined ones were made with 151 elements on each edge of the domain. The value of the diameter of the enrichment area was 0.30 .

\subsection{Results}

Let us recall that our choice of finite element scheme should lead to the following error estimates : $\left\|u^{h}-u\right\|_{H^{2}}=O(h)$ and $\left\|u^{h}-u\right\|_{L^{2}}=O\left(h^{2}\right)$, in the case of a regular problem (see Section 2). So, in the case of a cracked domain, a lack of accuracy is expected for a classical FEM, since the exact solution is not smooth enough. On the contrary, for both XFEM enrichment strategies, since the exact asymptotic displacement is added in the finite element basis, we expect to recover an optimal rate of convergence.

These considerations were confirmed by the numerical experiments. The convergence curves are presented on Fig. 11, for structured meshes. On these curves, one can also notice that the second enrichment strategy, named "Integral matching", is significantly more accurate than the first one.

XFEM achieve the optimal rate of convergence: it is close to 1 and 2 in $H^{2}$ and $L^{2}$ norms, respectively. The classical FEM shows a poorer accuracy, both in relative error and in rates of convergence. They are roughly divided by 2 , compared to the optimal ones. These convergence rates are presented in Tab. 1 . 

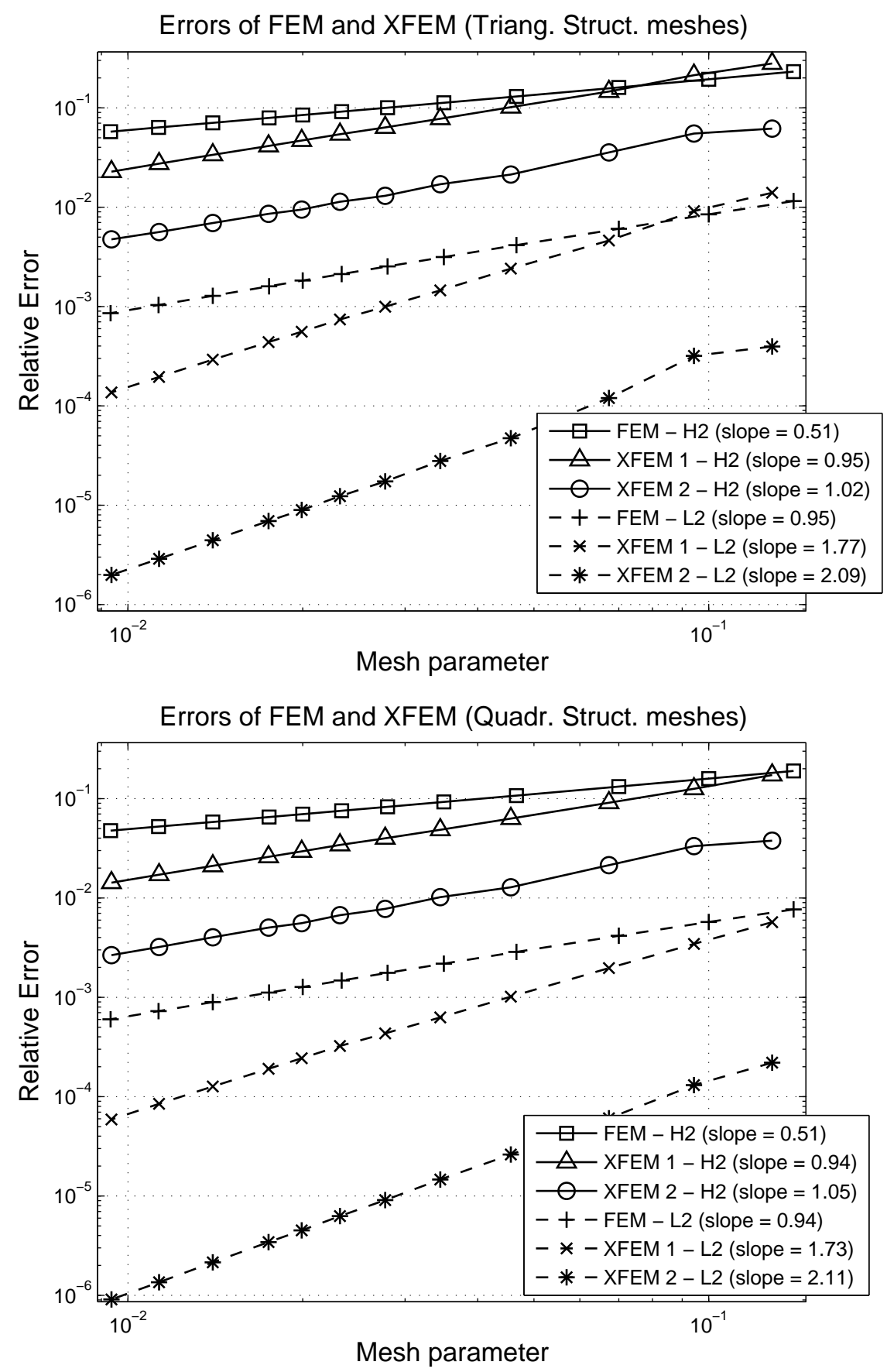

FIGURE 11. Convergence curves for the FEM and both XFEM strategies, on structured meshes. Top: triangular meshes. Bottom: quadrangular meshes.

\subsection{Robustness with respect to non-structured meshes}

The same experiment have been carried out on non-structured meshes. The results are presented Fig. 12. Here also, the second enrichment strategy is much more accurate than the first one, even if the slope of the first XFEM strategy is higher than the second one. The conclusion of the study is that the accuracy of the XFEM enrichment strategies does not decrease too much on non-structured meshes, the slope of first strategy is even higher on these non-structured meshes than on the structured ones. 


\begin{tabular}{|l|c|c|}
\hline & $H^{2}$ norm & $L^{2}$ norm \\
\hline FEM Tri. & 0.51 & 0.95 \\
\hline FEM Quad. & 0.51 & 0.94 \\
\hline XFEM 1 Tri. & 0.95 & 1.77 \\
\hline XFEM 1 Quad. & 0.94 & 1.73 \\
\hline XFEM 2 Tri. & 1.02 & 2.09 \\
\hline XFEM 2 Quad. & 1.05 & 2.11 \\
\hline
\end{tabular}

TABLE 1. Rates of convergence for FEM and XFEM, on structured meshes.

\section{Computation of Stress Intensity FaCtors}

In industrial applications dealing with cracked structures, the plate displacement is not straightforwardly meaningfull in terms of crack propagation. The Stress Intensity Factors (SIF) are linked to the energy release rate $G$, and they provide such information (we have $K_{1}^{2}+K_{2}^{2}$ proportional to $G$ ). However, the calculation of SIF usually needs the use of some specific post-treatment (computation of J-integral for instance).

An interesting feature of the integral matching methodology is that it provides a direct estimate of the SIF. Looking at the expressions of the asymptotic displacement (5) and at the numerical displacement (8), it appears that, if the method is convergent, the finite element coefficients $\left(c_{i}\right)$ should be close to $\left(K_{i}\right)$, up to a multiplicative constant. More precisely, we have:

$$
c_{i} \approx \frac{K_{i}\left(1-\nu^{2}\right)}{\sqrt{2} E \varepsilon(3+\nu)} .
$$

Let us remark that a similar idea has already been described and tested in a few works (see [14] for example).

So a numerical experiment of the method was carried out, for which the exact solution $u^{e x}$ was

$$
u^{e x}=u_{r}^{e x}+F_{1}+F_{2}
$$

where the regular part $u_{r}^{e x}$ is a fourth order polynomial $u_{r}^{e x}=x_{2}\left(1+10 x_{2}^{3}\right)$, and the singular $\left(F_{i}\right)$ functions are given by (7). Hence, the exact values of the SIF are set by the exact solution, and could be compared with the approximated values deduced from the $\left(c_{i}\right)$.

The material properties are the same as those indicated section 4.1. The numerical results are given Fig. 13. They show a low level of error: it is almost always lower than $5 \%$, and even most of the time lower than $1 \%$. This level of $5 \%$ seemed meaningful for us. In fact, in engineering applications, the computed SIF are used in a wider computation procedure, such as crack propagation, where other variables cannot be computed so precisely with respect to the exact physic of the problem. Moreover, even if the curves are quite oscillatory, a global decrease of the error can be observed.

\section{CONCLUDING REMARKS}

This paper presents an adaptation of XFEM to the Kirchhoff-Love plate model. The choice of this model allows to avoid the shear locking effect, which appears with the Mindlin-Reissner plate model (see [10]). Two different enrichment strategies are discussed and tested. The numerical results suggests that an optimal rate of convergence can be obtained for cracked plates despite the singularities. The second strategy appears to be better than the first one since the number of additional degrees of freedom is lower and the accuracy is better. Finally, the second strategy allows to obtain a direct computation of the stress intensity factors. A more complete study, including other numerical experiments, will be presented in a forthcoming paper [13]. 

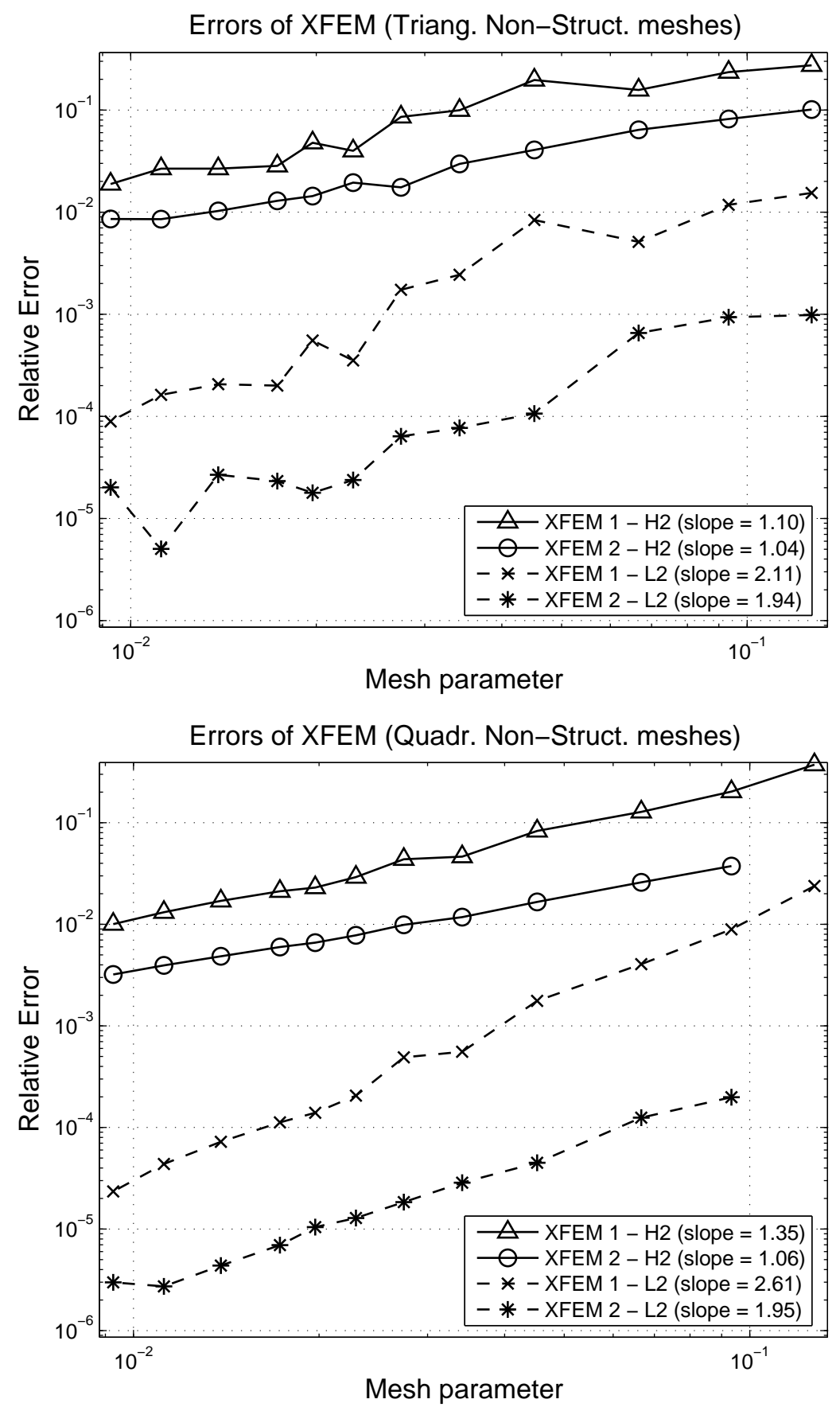

FIGURE 12. Convergence curves both XFEM strategies, on non-structured meshes. Top: triangular meshes. Bottom: quadrangular meshes.

\section{Acknowledgments}

This work was supported by a joint contract with Airbus and "Centre National de la Recherche Scientifique", and also supported by "Agence Nationale de la Recherche" (project ANR-05-JCJC-0182-01). The authors wish to thank Marc Balzano (Airbus France) and Patrick Laborde (Université Paul Sabatier) for their support all along this work. 

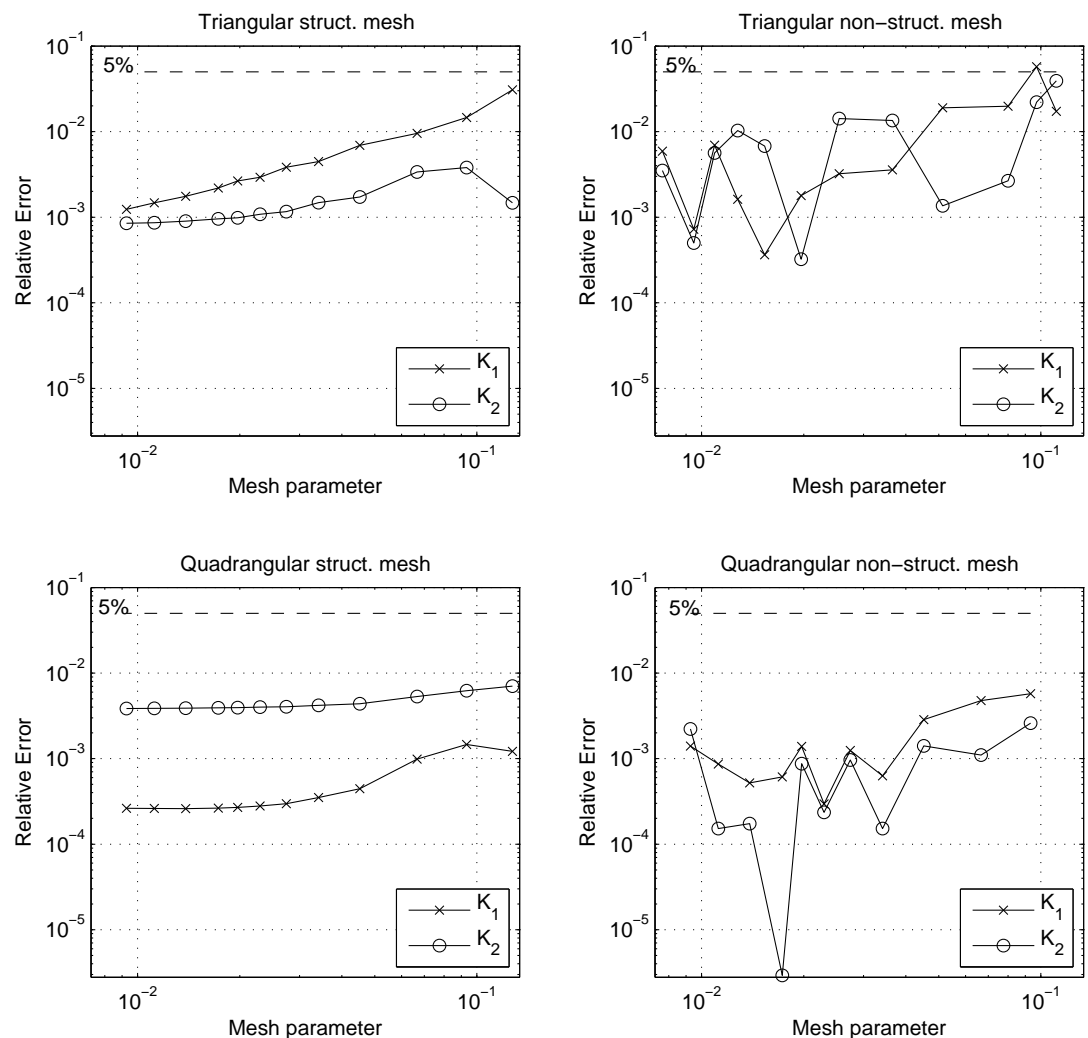

FIGURE 13. Relative error for the SIF computation.

\section{REFERENCES}

[1] R.A. Adams. Sobolev spaces. Academic Press, 1975.

[2] P.M.A. Areias, T. Belytschko. Analysis of three-dimensional crack initiation and propagation using the extended finite element method. Int. J. Numer. Meth. Engng., 63:760-788, 2005.

[3] E. Béchet, H. Minnebo, N. Moës, B. Burgardt. Improved implementation and robustness study of the X-FEM for stress analysis around cracks. Int. J. Numer. Meth. Engng., 64:1033-1056, 2005.

[4] S. Bordas, B. Moran. Enriched Finite Elements and Level Sets for Damage Tolerance Assessment of Complex Structures. Engng. Fract. Mech., 73:1176-1201, 2006.

[5] P.G. Ciarlet. The finite element method for elliptic problems. North-Holland, 1978.

[6] P. G. Ciarlet. Basic error estimates for elliptic problems. In "Handbook of numerical analysis, Vol. II", pages 17-351. NorthHolland, Amsterdam, 1991.

[7] P.G. Ciarlet, Ph. Destuynder. A justification of two-dimensionnal linear plate model. J. Mecan., Vol. 18, $\mathrm{n}^{\circ} 2,1979$.

[8] Ph. Destuynder. Une théorie asymptotique des plaques minces en élasticité linéaire. Masson, 1986.

[9] Ph. Destuynder, M. Djaoua. Sur une interprétation mathématique de l'intégrale de Rice en théorie de la rupture fragile. Mathematical Methods in the Applied Sciences, 3:70-87, 1981.

[10] J. Dolbow, N. Moës, T. Belytschko. Modeling fracture in Mindlin-Reissner plates with the extended finite element method. International Journal of Solids and Structures, 37:7161-7183, 2000.

[11] P. Grisvard. Singularities in boundary value problems. Masson, 1992.

[12] P. Laborde, J. Pommier, Y. Renard, M. Salaün. High order extended finite element method for cracked domains. Int. J. Numer. Meth. Engng., 64:354-381, 2005.

[13] J. Lasry, J. Pommier, Y. Renard, M. Salaün. eXtended Finite Element Methods for thin cracked plates, with Kirchhoff-Love theory. In preparation.

[14] X.Y. Liu, Q.Z. Xia, B.L. Karihaloo. XFEM for direct evaluation of mixed mode SIFs in homogeneous and bi-materials. Int. J. Numer. Meth. Engng., 59:1103-1118, 2004.

[15] R.H. McNeal. A simple quadrilateral shell element. Computer and Structures, 8:175-183, 1978. 
[16] N. Moës, T. Belytschko. XFEM : Nouvelles frontières pour les éléments finis. Revue européenne des éléments finis, 11:131-150, 1999.

[17] N. Moës, J. Dolbow, T. Belytschko. A finite element method for crack growth without remeshing. Int. J. Numer. Meth. Engng., 46:131-150, 1999

[18] N. Moës, A. Gravouil, T. Belytschko. Non-planar 3D crack growth by the extended finite element and level sets, Part I: Mechanical model. Int. J. Numer. Meth. Engng., 53:2549-2568, 2002.

[19] J. Pommier, Y. Renard. Getfem++, an open source generic C++ library for finite element methods. http://home.gna.org/getfem/

[20] N. Sukumar, Z.Y. Huang, J.-H. Prévost, Z. Suo. Partition of unity enrichment for bimaterial interface cracks. Int. J. Numer. Meth. Engng., 59:1075-1102, 2004.

[21] A. Zucchini, C. Y. Hui, A. T. Zehnder Crack tip stress fields for thin, cracked plates in bending, shear and twisting: A comparison of plate theory and three-dimensional elasticity theory solutions. Int. J. of Fracture, 104:387-407, 2000. 